\title{
Microscopic view on Landau level broadening mechanisms in graphene
}

\author{
Hannah Funk and Andreas Knorr \\ Institute of Theoretical Physics, Nonlinear Optics and Quantum Electronics, Technical University Berlin, Germany
}

Florian Wendler* and Ermin Malic

Department of Applied Physics, Chalmers University of Technology, Gothenburg, Sweden

(Received 25 August 2015; revised manuscript received 23 October 2015; published 24 November 2015)

\begin{abstract}
Placing a two-dimensional sheet of graphene in an external magnetic field the continuous electronic band structure is discretized due to Landau quantization. The resulting optical transitions are subject to a broadening, which can lead to a significant overlap of Landau levels. We investigate the possible microscopic processes that could cause a broadening of the corresponding peaks in the absorption spectrum of Landau-quantized graphene: (i) radiative decay, (ii) Coulomb interaction, (iii) optical phonons, (iv) acoustic phonons, and (v) impurities. Since recent experiments have shown that independent of the magnetic field the resolvable number of Landau levels is constant, we put a special focus on the dependence of the broadening on the external magnetic field $B$ and the Landau level index $n$. Our calculations reveal the impurities to be the crucial broadening mechanism, where different regimes of well separated and densely spaced Landau levels need to be taken into account. Furthermore, carrier-carrier and carrier-phonon scattering give rise to a very specific dependence on the Landau level index $n$ that has not been observed yet.
\end{abstract}

DOI: 10.1103/PhysRevB.92.205428

PACS number(s): 42.25.Bs, 78.67.Wj, 73.22.Pr, 76.40.+b

\section{INTRODUCTION}

In an external magnetic field, the electronic band structure of graphene splits up into nonequidistant Landau levels (LLs) displaying the relativistic quantum Hall effect $[1,2]$. Due to the rather unusual Landau level spectrum [3] with $\epsilon_{n}= \pm \hbar \omega_{c} \sqrt{n}$, where $\omega_{c}$ is the cyclotron frequency and $n$ the Landau level index, the carrier dynamics in graphene subject to a magnetic field show some remarkable features [4-6] and have started to attract considerable attention in current research [4-13].

In practice, optical transitions between discrete energy levels are always subject to a finite broadening, which appears as a consequence of interactions with the environment or even with the light field in vacuum. This is of special importance in Landau-quantized graphene, where carrier-carrier as well as carrier-phonon scattering sensitively depend on the magnitude of the LL broadening [13]. Furthermore, the broadening, if large enough, leads to a significant overlap of LLs and thereby counteracts the quantization. The inset in Fig. 1 shows the original Dirac cone and the energetically lowest discrete LLs with indices $n=0,1,2,3,4$ in the conduction and the valence band, illustrating that the spacing between adjacent LLs decreases with increasing $n$. The LL spacing is determined by the cyclotron frequency $\omega_{c}=v_{\mathrm{F}} \sqrt{2 e_{0} B / \hbar}$, where $\hbar \omega_{c}$ corresponds to the energy difference between $\mathrm{LL}_{+1}$ and $\mathrm{LL}_{0}$. Since it is proportional to $\sqrt{B}$, one could assume that the number of well-separated and experimentally resolvable LLs increases at larger magnetic fields $B$. However, in a recent experiment by Orlita et al. [14], the number of resolvable LLs was found to be constant for different magnetic field strengths suggesting a $\sqrt{n B}$ dependence of the LL broadening that exactly cancels the increased LL spacing.

In this article, we study different microscopic processes contributing to the Landau level broadening focusing in

\footnotetext{
*florian.wendler@chalmers.se
}

particular on their $n$ and $B$ dependence. We take into account impurity-induced LL broadening as well as broadening due to radiative damping, carrier-carrier, and carrier-phonon scattering. We calculate the absorption spectrum of Landauquantized graphene based on a microscopic theory and analyze the impact of these broadening mechanisms on the absorption peaks. The latter correspond to inter-LL transitions obeying the optical selection rules $\mathrm{LL}_{n} \rightarrow \mathrm{LL}_{n \pm 1}$ [15-17]. Figure 1 illustrates the absorption spectrum of intrinsic graphene (zero doping) under Landau quantization with a broadening $\Gamma_{n, n \pm 1}$.

\section{THEORETICAL APPROACH}

In this section, we first introduce the many-particle Hamilton operator of our system, before we calculate the absorption spectrum of Landau-quantized graphene.

\section{A. Many-particle Hamilton operator}

The Hamilton operator reads in second quantization:

$$
\hat{H}=\underbrace{\hat{H}_{0, \mathrm{el}}+\hat{H}_{0, \mathrm{ph}}}_{\hat{H}_{0}}+\underbrace{\hat{H}_{\mathrm{el}-\text { light }}+\hat{H}_{\mathrm{el}-\mathrm{el}}+\hat{H}_{\mathrm{el} \text {-ph }}+\hat{H}_{\mathrm{el}-\mathrm{imp}}}_{\hat{H}_{\text {int }}}
$$

and consists of the free contribution of electrons and phonons as well as the electron-light, electron-electron, electronphonon, and electron-impurity interactions.

(a) Free contribution. The contribution of the free electron kinetic energy reads

$$
\hat{H}_{0, \mathrm{el}}=\sum_{i} \varepsilon_{i} a_{i}^{\dagger} a_{i}
$$

with the electronic dispersion $\varepsilon_{i}=\lambda_{i} v_{\mathrm{F}} \sqrt{2 \hbar e_{0} n_{i} B}$ that is calculated with tight-binding wave functions in combination with the Peierls substitution considering the presence of an external magnetic field [3,13]. It depends on the Fermi velocity 


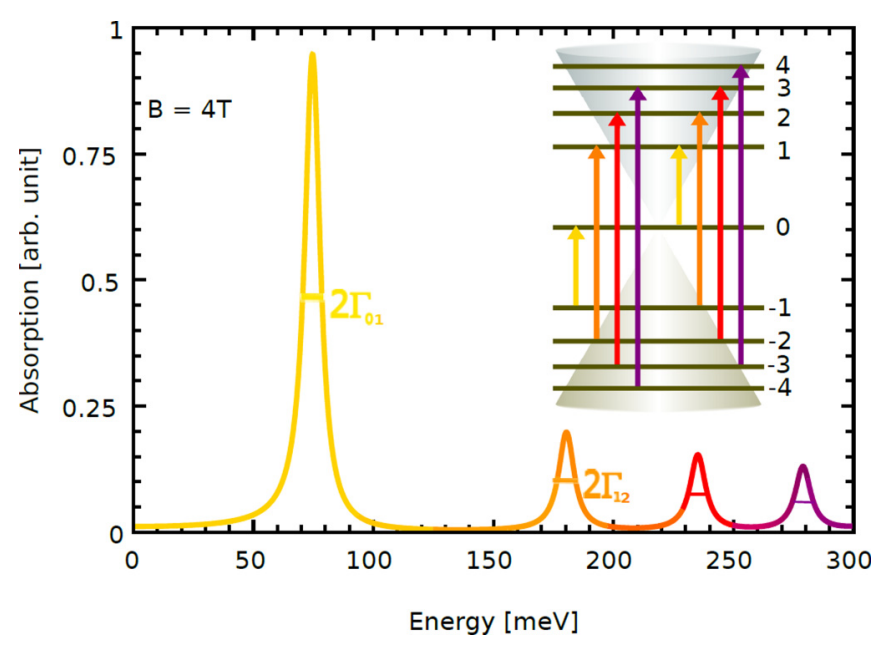

FIG. 1. (Color online) Spectrum of Landau-quantized graphene for the first four interband transitions at an external magnetic field of $4 \mathrm{~T}$. The corresponding transition between the discretized Landau levels are shown in the inset with the Dirac cone in the background. Here, $\Gamma_{10}$ denotes the broadening of the transition $\mathrm{LL}_{0} \rightarrow \mathrm{LL}_{+1}, \Gamma_{12}$ the transition $\mathrm{LL}_{-1} \rightarrow \mathrm{LL}_{+2}$, etc. The full width at half maximum $(\mathrm{FWHM})$ equals $\Gamma_{\mathrm{FWHM}}=2 \Gamma$.

$v_{\mathrm{F}}$, the magnetic field $B$, and the indices $\lambda$ distinguishing the conduction $(\lambda=+1)$ from the valence band $(\lambda=-1)$ and $n$ denoting the Landau levels. The energy does not depend on the spin $(s)$, the valley $(\xi)$, nor on the position of the electron in the two-dimensional graphene plane, which is connected to the quantum number [3] $m$. Therefore, the full quantum state is defined by the compound index $i=\{\xi, \lambda n, m, s\}$ and every Landau level $\mathrm{LL}_{\lambda n}$ is highly degenerate.

The kinetic energy of free phonons describing the vibrations of lattice ions around their equilibrium positions is described by

$$
\hat{H}_{0, \mathrm{pn}}=\sum_{\mathbf{q}, \kappa} \varepsilon_{\mathbf{q} \kappa}\left(b_{\mathbf{q} \kappa}^{\dagger} b_{\mathbf{q} \kappa}+\frac{1}{2}\right),
$$

where $\varepsilon_{\mathbf{q} \kappa}=\hbar \omega_{\mathbf{q} \kappa}$ indicates the dispersion of the phonons and $b_{\mathbf{q} \kappa}^{\dagger}$ and $b_{\mathbf{q} \kappa}$ are the creation and annihilation operators acting on the phonon state which consists of the quasimomentum $\mathbf{q}$ and the branch index $\kappa=\mathrm{LO}, \mathrm{LA}, \mathrm{TO}, \mathrm{TA}, \mathrm{ZO}$, where $\mathrm{L}$ are longitudinal, $\mathrm{T}$ transverse, $\mathrm{Z}$ flexural, $\mathrm{O}$ optical (out-of-phase oscillation of neighboring lattice atoms), and A acoustic (inphase oscillation) phonon modes.

(b) Carrier-light interaction. To calculate the absorption in Sec. II B we use the semiclassical approach for the coupling of electrons with the light. Later on in this section, we will also apply the fully quantum mechanical approach to determine the radiative broadening of Landau levels.

The semiclassical electron-light interaction Hamiltonian reads

$$
\hat{H}_{\mathrm{el}-\mathrm{light}}^{\mathrm{sc}}=i \hbar \frac{e_{0}}{m_{0}} \sum_{i j} \mathbf{M}_{i j} \cdot \mathbf{A}(t) a_{i}^{\dagger} a_{j},
$$

where $e_{0}$ is the charge and $m_{0}$ the mass of a free electron, $\mathbf{A}(t)$ is the vector potential of the optical field, and $\mathbf{M}_{i j}=$ $\left\langle\Psi_{i}(\mathbf{r})|\nabla| \Psi_{j}(\mathbf{r})\right\rangle$ is the optical matrix element. Here, the excitation pulse is modeled using a Gaussian envelope function and consists of two contributions stemming from left $\left(\sigma^{+}\right)$and right $\left(\sigma^{-}\right)$circularly polarized light:

$$
\mathbf{A}(t)=e^{-\frac{t^{2}}{2 \sigma^{2}}}\left[A_{0}^{\sigma^{+}}\left(\begin{array}{c}
\cos \left(\omega_{L} t\right) \\
\sin \left(\omega_{L} t\right)
\end{array}\right)+A_{0}^{\sigma^{-}}\left(\begin{array}{c}
\cos \left(\omega_{L} t\right) \\
-\sin \left(\omega_{L} t\right)
\end{array}\right)\right] .
$$

The corresponding prefactors $A_{0}^{\sigma^{ \pm}}=\frac{1}{\omega_{L}} \sqrt{\frac{\varepsilon_{\mathrm{pf}}}{\sqrt{\pi} \varepsilon_{0} c \sigma_{t}}}$ contain the width of the pulse $\sigma_{t}$, the pump fluence $\varepsilon_{\mathrm{pf}}$, and the frequency of the light pulse $\omega_{L} \approx \omega_{i j}=\left(\varepsilon_{i}-\varepsilon_{j}\right) / \hbar$, which is assumed to be in resonance with an inter-Landau level transition. In the basis of the Jones vectors [18] $\hat{\boldsymbol{\varepsilon}}^{ \pm}=\frac{1}{\sqrt{2}}\left(\begin{array}{c}1 \\ \mp i\end{array}\right)$, the optical excitation pulse reads

$$
A^{ \pm}(t)=e^{-\frac{t^{2}}{2 \sigma^{2}}}\left(A_{0}^{\sigma^{+}} \frac{1}{\sqrt{2}} e^{\mp i \omega_{i j} t}+A_{0}^{\sigma^{-}} \frac{1}{\sqrt{2}} e^{ \pm i \omega_{i j} t}\right) .
$$

The characteristic optical selection rules $\mathrm{LL}_{n} \rightarrow \mathrm{LL}_{n \pm 1}$ of Landau-quantized graphene are inherent to its optical matrix element $[4,19]$ :

$$
\begin{aligned}
\mathbf{M}_{i, j}= & -\mathbf{M}_{i, j}^{*} \\
= & i \delta_{\xi_{i}, \xi_{j}} \delta_{m_{i}, m_{j}} \delta_{s_{i}, s_{j}} \frac{\alpha_{n_{i}} \alpha_{n_{j}} m_{0} v_{\mathrm{F}}}{2 \sqrt{2} \hbar} \\
& \times\left(\lambda_{i} \delta_{n_{i}, n_{j}+1} \hat{\boldsymbol{\varepsilon}}^{+}+\lambda_{j} \delta_{n_{i}, n_{j}-1} \hat{\boldsymbol{\varepsilon}}^{-}\right),
\end{aligned}
$$

with the constants $\alpha_{n=0}=\sqrt{2}, \alpha_{n \neq 0}=1$.

To calculate the radiative broadening in Sec. IIIB we treat the interaction of electrons with the light field quantum mechanically. To this end, the radiation is quantized by expanding the vector potential in plane waves [20]. The electron-photon Hamiltonian then depends on the ladder operators of the photons $c_{\mathbf{q}}^{\dagger}$ and $c_{\mathbf{q}}$ :

$$
\hat{H}_{\mathrm{el}-\text { light }}^{\mathrm{qm}}=\hbar \sum_{i, j} \sum_{\mathbf{q}, \sigma} \tilde{M}_{\mathbf{q}, \sigma}^{i j} a_{i}^{\dagger} a_{j}\left(c_{\mathbf{q}, \sigma}^{\dagger}+c_{\mathbf{q}, \sigma}\right),
$$

and the optical matrix element reads

$$
\tilde{M}_{\mathbf{q}, \sigma}^{i j}=-i \frac{e_{0}}{m_{0}} \sqrt{\frac{\hbar}{2 \omega_{\mathbf{q}} \varepsilon_{0} V}} \frac{1}{\sqrt{2}} \hat{\boldsymbol{\varepsilon}}^{\sigma} \cdot \underbrace{\left\langle\Psi_{i}(\mathbf{r})\left|e^{i \mathbf{q r}} \nabla\right| \Psi_{j}(\mathbf{r})\right\rangle}_{\approx\left\langle\Psi_{i}(\mathbf{r})|\nabla| \Psi_{j}(\mathbf{r})\right\rangle=\mathbf{M}_{i j}},
$$

with the frequency $\omega_{q}$ of a photon in the mode given by the wave vector $\mathbf{q}$ and the polarization vector for circularly polarized light $\hat{\boldsymbol{\varepsilon}}^{\sigma}$ corresponding to a Jones vector $\hat{\boldsymbol{\varepsilon}}^{ \pm}$. The latter depends on the polarization of the excitation pulse (leftor right-hand circularly polarized). Since the wavelength of light is much larger than the lattice constant of graphene, we can apply the dipole approximation by retaining only the first term in the Taylor series of $e^{i \mathbf{q r}} \approx 1$.

(c) Carrier-carrier interaction. The Coulomb interaction is described by

$$
\hat{H}_{\mathrm{el}-\mathrm{el}}=\frac{1}{2} \sum_{\mathrm{abcd}} \mathbf{V}_{\mathbf{c d}}^{\mathbf{a b}} a_{a}^{+} a_{b}^{+} a_{c} a_{d}
$$


where electrons in the states $c, d$ exchange energy and momentum and scattering to the states $a, b$. The strength of the interaction is determined by the Coulomb matrix element in the presence of an external magnetic field [4]:

$$
\begin{aligned}
\mathbf{V}_{\mathbf{c d}}^{\mathbf{a b}}= & \alpha_{n_{a}} \alpha_{n_{b}} \alpha_{n_{c}} \alpha_{n_{d}} \frac{e_{0}^{2} \delta_{\xi_{a} \xi_{c}} \delta_{\xi_{b} \xi_{d}}}{8 \pi^{2} \varepsilon_{0} \varepsilon_{r}} \int_{0}^{\infty} d q \int_{0}^{2 \pi} d \varphi \\
& \times\left[\lambda_{a} \lambda_{c} \mathscr{F}_{n_{c}-1, m_{c}}^{n_{a}-1, m_{a}}(\mathbf{q})+\mathscr{F}_{n_{c}, m_{c}}^{n_{a}, m_{a}}(\mathbf{q})\right] \\
& \times\left[\lambda_{b} \lambda_{d} \mathscr{F}_{n_{d}-1, m_{d}}^{n_{b}-1, m_{b}}(-\mathbf{q})+\mathscr{F}_{n_{d}, m_{d}}^{n_{b}, m_{b}}(-\mathbf{q})\right],
\end{aligned}
$$

with the relative and vacuum permittivities $\varepsilon_{r}$ and $\varepsilon_{0}$, the momentum expressed in polar coordinates $\mathbf{q}=(q, \varphi)$, and the form factor describing the geometric structure of graphene [13]:

$$
\begin{aligned}
& \mathcal{F}_{n, m}^{n^{\prime} m^{\prime}}(\mathbf{q})=(-1)^{\Theta\left( \pm n^{\prime} \mp n\right)\left|n-n^{\prime}\right|+\Theta\left( \pm m^{\prime} \mp m\right)\left|m-m^{\prime}\right|} e^{-\frac{\hbar q^{2}}{2 e_{0} B}} \\
& \times \sqrt{\frac{\min \left(m^{\prime}, m\right) !}{\max \left(m^{\prime}, m\right) !}} \sqrt{\frac{\min \left(n^{\prime}, n\right) !}{\max \left(n^{\prime}, n\right) !}}\left(q \sqrt{\frac{\hbar}{2 e_{0} B}}\right)^{\left|m-m^{\prime}\right|+\left|n-n^{\prime}\right|} \\
& \times\left(e^{i \varphi}\right)^{m-m^{\prime}-n+n^{\prime}} L_{\min \left(m^{\prime}, m\right)}^{\left|m-m^{\prime}\right|}\left(\frac{\hbar q^{2}}{2 e_{0} B}\right) L_{\min \left(n^{\prime}, n\right)}^{\left|n-n^{\prime}\right|}\left(\frac{\hbar q^{2}}{2 e_{0} B}\right),
\end{aligned}
$$

with the associated Laguerre polynomials $L_{n}^{\alpha}(x)$.

(d) Carrier-phonon interaction. The Hamiltonian of the electron-phonon interaction reads

$$
\hat{H}_{\mathrm{el}-\mathrm{pn}}=\sum_{i, j} a_{i}^{\dagger} a_{j} \sum_{\mathbf{q} \kappa} g_{i j}^{\mathbf{q} \kappa}\left(b_{\mathbf{q} \kappa}+b_{\mathbf{q} \kappa}^{\dagger}\right)
$$

with the electron-phonon matrix element $g_{i j}^{\mathbf{q} \kappa}=\left\langle\Psi_{i}(\mathbf{r})\right|$ $\mathbf{V}_{\mathrm{ph}}^{\mathbf{q} \kappa}(\mathbf{r})\left|\Psi_{j}(\mathbf{r})\right\rangle$ that is determined by the coupling-potential $\mathbf{V}_{\mathrm{ph}}^{\mathbf{q} \kappa}$. The diagonal elements of $\mathbf{V}_{\mathrm{ph}}^{\mathbf{q} \kappa}$ represent on-site coupling considered via a deformation potential, whereas the offdiagonal elements describe the coupling of neighboring sites by modulated hopping [13,21,22].

In this work, we consider single-phonon processes. Flexural modes, which are only excited in pairs [23], are thus omitted. In the case of long-wavelength acoustic phonons, the on-site coupling is one magnitude larger than the off-site coupling [22]; hence we concentrate on the deformation potential, where the main contribution stems from the $\Gamma \mathrm{LA}$ mode. In contrast, the optical modes strongly couple via the modulated hopping mechanism and are considered at the $\Gamma$ and $\mathrm{K}$ point. For a transition $j \rightarrow i$ the coupling matrix elements for electrons with acoustic and optical phonons in Landau-quantized graphene then reads $[9,13]$

$$
\begin{aligned}
g_{i j}^{\mathbf{q} L A}= & i D|\mathbf{q}| \delta_{\xi_{i} \xi_{j}} \alpha_{n_{i}} \alpha_{n_{j}} \sqrt{\frac{\hbar}{4 M A \omega_{\mathbf{q} L A}}} \\
& \times\left(\lambda_{i} \mathscr{F}_{n_{i}-1, m_{i}}^{n_{j}, m_{j}}(-\mathbf{q})+\lambda_{j} \mathscr{F}_{n_{i}, m_{i}}^{n_{j}-1, m_{j}}(\mathbf{q})\right),
\end{aligned}
$$

$$
\begin{aligned}
g_{i j}^{\mathbf{q} \Gamma-O}= & \frac{3 \alpha_{n_{i}} \alpha_{n_{j}} \beta \hbar^{2} v_{F}}{\sqrt{2 M A \varepsilon_{\mathbf{q}, \Gamma-O}} a_{0}^{2}} \\
& \times\left(\lambda_{i} \mathscr{F}_{n_{i}-1, m_{i}}^{n_{j}, m_{j}}(-\mathbf{q}) \pm \lambda_{j} \mathscr{F}_{n_{i}, m_{i}}^{n_{j}-1, m_{j}}(\mathbf{q})\right), \\
g_{i j}^{\mathbf{q} K-O}= & \alpha_{n_{i}} \alpha_{n_{j}} \sqrt{\left\langle g_{K-O}^{2}\right\rangle_{\mathrm{DFT}}} \\
& \times\left(\lambda_{i} \mathscr{F}_{n_{i}-1, m_{i}}^{n_{j}, m_{j}}(-\mathbf{q})-\lambda_{j} \mathscr{F}_{n_{i}, m_{i}}^{n_{j}-1, m_{j}}(\mathbf{q})\right),
\end{aligned}
$$

where $\omega_{\mathbf{q}, \mathrm{LA}}=v_{\mathrm{F}}|\mathbf{q}|$ is the dispersion of acoustic phonons [24], $M=7.6 \times 10^{-8} \mathrm{~g} \mathrm{~cm}^{-2}$ the graphene mass density [25], $A$ the area of graphene, $D=16 \mathrm{eV}$ the deformation potential [26], $\beta \approx 2$ the coupling parameter [27], and $\varepsilon_{\mathbf{q}, \Gamma-O}$ the optical phonon energy. Furthermore, the sign \pm in Eq. (15) corresponds to the modes $\Gamma$ TO and $\Gamma \mathrm{LO}$, respectively, and the carrier-phonon interaction strength $\left\langle g_{K-T O}^{2}\right\rangle_{\mathrm{DFT}}=$ $0.0994 \mathrm{eV}^{2} A_{u c} / A$ and $\left\langle g_{K-L O}^{2}\right\rangle_{\mathrm{DFT}}=0.00149 \mathrm{eV}^{2} A_{u c} / A$ is based on numerical calculations by Piscanec et al. [28]. Note that the coupling strength is two orders of magnitude smaller for KLO phonons in comparison to the other optical phonons; thus their impact to the energy broadening is comparatively small.

(e) Carrier-impurity interaction. The impurities are assumed to be randomly distributed scatterers at carbon atom sites resulting in an effective potential [29-32]. In agreement with experimental results, we consider short-range scatterers [14]. The interaction of electrons with impurities in the sample is determined by the Hamilton operator:

$$
\hat{H}_{\mathrm{el-imp}}=\sum_{i j} D_{i j} a_{i}^{\dagger} a_{j} .
$$

In the appearing matrix element $D_{i j}=\left\langle\Psi_{i}(\mathbf{r})|U(\mathbf{r})| \Psi_{j}(\mathbf{r})\right\rangle$ the impurities are represented by the Gaussian white noise potential $U(\mathbf{r})$ [33]. The impurity average of a single impurity potential vanishes, i.e., $\overline{D_{12}}=0$, while $\overline{D_{12} D_{34}}=\delta_{\xi_{1}, \xi_{2}} \delta_{\xi_{3}, \xi_{4}} \frac{\hbar^{2} v_{F}^{2}}{l_{B}^{2} A_{\text {imp }}} \mathscr{F}_{n_{1} n_{2} n_{3} n_{4}}^{m_{1} m_{2} m_{3} m_{4}}$ depends on the form factor $\mathscr{F}_{n_{1} n_{2} n_{3} n_{4}}^{m_{1} m_{2} m_{4}}$, which can be found in the Supplemental Material of Ref. [4]. Furthermore, the dimensionless parameter $A_{\text {imp }}$ enters and characterizes the scattering strength. It is proportional to the number of scatterers in the sample and their effective potential [29] and is adjusted to experimental observations on LL broadening $(A=255)$. More details on how the impurity-induced LL broadening has been accounted for in the well separated and densely spaced LL regime is discussed in Sec. III A.

Note that we do not take geometrical confinement effects into account that compete with the confinement of electrons into cyclotron orbits [34]. Assuming a magnetic length scale much smaller than the geometrical confinement, they are negligible [35].

\section{B. Absorption spectra}

In linear optics, the absorption coefficient $\tilde{\alpha}$ at frequency $\omega$ is determined by the imaginary part of the optical susceptibility [36] $\chi(\omega)$. For atomically thin two-dimensional materials, 
such as graphene, we can introduce the Taylor approximated absorbance $\alpha(\omega)=1-e^{-L_{z} \tilde{\alpha}(\omega)} \approx L_{z} \tilde{\alpha}(\omega)$ as

$$
\alpha_{ \pm}(\omega)=\frac{\omega L_{z}}{n(\omega) c} \operatorname{Im}\left\{\chi_{ \pm}(\omega)\right\},
$$

with the graphene layer thickness $L_{z}$, the speed of light $c$, and the refractive index $n(\omega)=1$ in vacuum. The subscript \pm denotes the $\sigma^{ \pm}$-circularly polarized components for all respective quantities [cf. Eq. (6)] and will be used throughout the paper.

The optical susceptibility $\chi(\omega)$ can be expressed through the current density $\mathbf{j}(\omega)$ and the vector potential of the light field $\mathbf{A}(\omega)$ [37]:

$$
\chi_{ \pm}(\omega)=\frac{j_{ \pm}(\omega)}{\varepsilon_{0} \omega^{2} A_{ \pm}(\omega)},
$$

where $\varepsilon_{0}$ is the vacuum permittivity and the current density in Landau-quantized graphene reads [37]

$$
j_{ \pm}(\omega)=\frac{-i \hbar e_{0}}{2 m_{0} A L_{z}} \sum_{i j}\left[M_{i j}^{ \pm} p_{i j}(\omega)+M_{j i}^{ \pm} p_{j i}(\omega)\right],
$$

with the extent of graphene in the $z$-direction $L_{z}$.

To determine the absorbance, we calculate the microscopic polarization $p_{i j}(t)=\left\langle a_{i}^{\dagger} a_{j}\right\rangle$ entering in Eq. (20) by deriving Bloch equations for Landau-quantized graphene [5,36,37]:

$$
\begin{gathered}
\dot{\rho}_{i}(t)=2 \sum_{l} \operatorname{Re}\left\{\Omega_{i l}(t) p_{i l}(t)\right\}+S_{i}^{\text {in }}(t)\left(1-\rho_{i}(t)\right) \\
\quad-S_{i}^{\text {out }}(t) \rho_{i}(t), \\
\dot{p}_{i j}(t)=i \omega_{i j} p_{i j}(t)+\Omega_{j i}(t)\left(\rho_{i}(t)-\rho_{j}(t)\right)-\frac{\Gamma_{i j}}{\hbar} p_{i j},
\end{gathered}
$$

where $\Omega_{i j}=e_{0} / m_{0} \mathbf{M}_{i j} \cdot \mathbf{A}$ is the Rabi frequency containing the optical matrix element $\mathbf{M}_{i j}$ and the vector potential $\mathbf{A}$. The equations describe the coupled dynamics between $p_{i j}(t)$ and $\rho_{i}(t)=\left\langle a_{i}^{\dagger} a_{i}\right\rangle$, the latter corresponding to the carrier occupation $\rho_{i}(t)=\left\langle a_{i}^{\dagger} a_{i}\right\rangle$ in the state $i$. In the case of linear optics, we assume a constant carrier occupation $\rho\left(\varepsilon_{n}^{\lambda}\right)$ given by the Fermi function. The time-dependent Coulomb- and phonon-induced scattering rates $S_{i}^{\text {in/out }}(t)=S_{i_{\mathrm{el}}}^{\text {in/out }}(t)+S_{i_{\mathrm{ph}}}^{\text {in/out }}(t)$ in Eq. (21) are important to study the many-particle induced broadening mechanisms for Landau levels and will be further described in Secs. III C and III D 1.

The dephasing of the microscopic polarization is determined by

$$
\Gamma_{i j}=\Gamma_{i j}^{\mathrm{rad}}+\Gamma_{i j}^{\mathrm{Coul}}+\Gamma_{i j}^{\mathrm{ph}-\mathrm{op}}+\Gamma_{i j}^{\mathrm{imp}} .
$$

It comprises all different scattering channels that contribute to the Landau level broadening (cf. Sec. III): (i) radiative broadening $\Gamma_{i j}^{\mathrm{rad}}$, (ii) Coulomb-induced broadening $\Gamma_{i j}^{\text {Coul }}$, (iii) broadening due to the scattering with optical phonons $\Gamma_{i j}^{\mathrm{Ph} \text {-op }}$, and (iv) impurity-induced broadening $\Gamma_{i j}^{\mathrm{imp}}$. The contribution of acoustic phonons is included nonperturbatively within the independent boson model (IBM) [38]: cf. the discussion below. The investigated broadening mechanisms can depend on the Landau level index $n$, the external magnetic field $B$, and the temperature $T$.
Applying the Fourier transform of Eq. (22) and using Eqs. (18)-(20), the absorbance reads

$$
\begin{aligned}
\alpha_{ \pm}(\omega)= & \sum_{i j} \frac{\left(\alpha_{n_{i}} \alpha_{n_{j}} v_{\mathrm{F}}\right)^{2} e_{0}^{3} B}{8 \pi \hbar c \varepsilon_{0} n(\omega) \omega} \delta_{n_{j}, n_{i} \pm 1}\left[\frac{\Gamma_{i j}}{\Gamma_{i j}^{2}+\hbar^{2}\left(\omega-\omega_{i j}\right)^{2}}\right. \\
& -\underbrace{\frac{\Gamma_{i j}}{\Gamma_{i j}^{2}+\hbar^{2}\left(\omega+\omega_{i j}\right)^{2}} \frac{A^{\mp}(\omega)}{A^{ \pm}(\omega)}}_{\text {neglegtet in RWA }}]
\end{aligned}
$$

Here, we performed the sum over the valley, spin, and $m$ degrees of freedom resulting in the prefactor $2 L^{2} e_{0} B /(\pi \hbar)$. The Kronecker $\delta$ 's describe the optical selection rules and stem from Eq. (7). When pumping with circular polarized radiation, the second term proportional to $\frac{A^{\mp}(\omega)}{A^{ \pm}(\omega)} \sim e^{ \pm i 2 \omega_{i j} t}$ can be neglected according to the rotating wave approximation (RWA).

Evaluating Eq. (24), we have microscopic access to the absorption spectrum of Landau-quantized graphene exhibiting pronounced peaks for the optically excited inter-LL transitions. Within the RWA, $\sigma^{+}$-polarized radiation excites transitions with $n \rightarrow n+1$, while $\sigma^{-}$-polarized light induces transitions with $n \rightarrow n-1$ [39]. The absorption peaks have a Lorentzian shape in Eq. (24) with the broadening $\Gamma_{i j}$, while their position is determined by the respective transition energy $\left(\varepsilon_{i}-\varepsilon_{j}\right)=\hbar \omega_{i j}$.

Independent boson model (IBM). To incorporate the influence of acoustic phonons on the broadening of LL peaks in absorption spectra, we analyze the effect of nonenergy conserving (non-Markovian) processes occurring on very short time scales within the time-energy uncertainty. The reason is that the energy of the acoustic phonons is too small to efficiently induce inter-LL transitions [4]. For this purpose, we apply the independent boson model, which is exactly solvable [38] for a two-level system with a bosonic bath.

In the regime of pure dephasing, the radiation causes a polarization of the material, but does not change the carrier occupation. Considering a system that has been excited before $t=0$, we solely deal with the interaction of the system with phonons for $t \geqslant 0$. In contrast to the correlation expansion used in Eqs. (21) and (22) we apply here the Wick's theorem [40], which is applicable in the interaction picture of quantum mechanics and allows one to simplify an arbitrary product of creation and annihilation operators by reducing it to sums of products of pairs of these operators. The polarization $p_{i j}(t)=\left\langle a_{i}^{\dagger} a_{j}\right\rangle$ is obtained in the interaction picture where the dynamics is determined by the time evolution operator $U\left(t, t_{0}\right)$ :

$$
p_{i j}(t)=\left\langle U^{\dagger}\left(t, t_{0}\right)\left(a_{i}^{\dagger} a_{j}\right)\left(t_{0}\right) U\left(t, t_{0}\right)\right\rangle .
$$

To deduce the absorbance [Eq. (18)], we perform a Fourier transformation of the microscopic polarization, insert it into Eq. (20) for the current, and obtain

$$
\begin{aligned}
\alpha_{ \pm}^{\mathrm{IBM}}(\omega)= & \sum_{i j} \frac{\left(\alpha_{n_{i}} \alpha_{n_{j}} v_{\mathrm{F}}\right)^{2} e_{0}^{3} B}{8 \pi \hbar^{2} c \varepsilon_{0} n(\omega) \omega^{2}} \delta_{n_{j}, n_{i} \pm 1} \\
& \times \operatorname{Re}\left\{\int_{0}^{\infty} e^{X^{i j}(t)} e^{-i \omega t} d t\right\},
\end{aligned}
$$




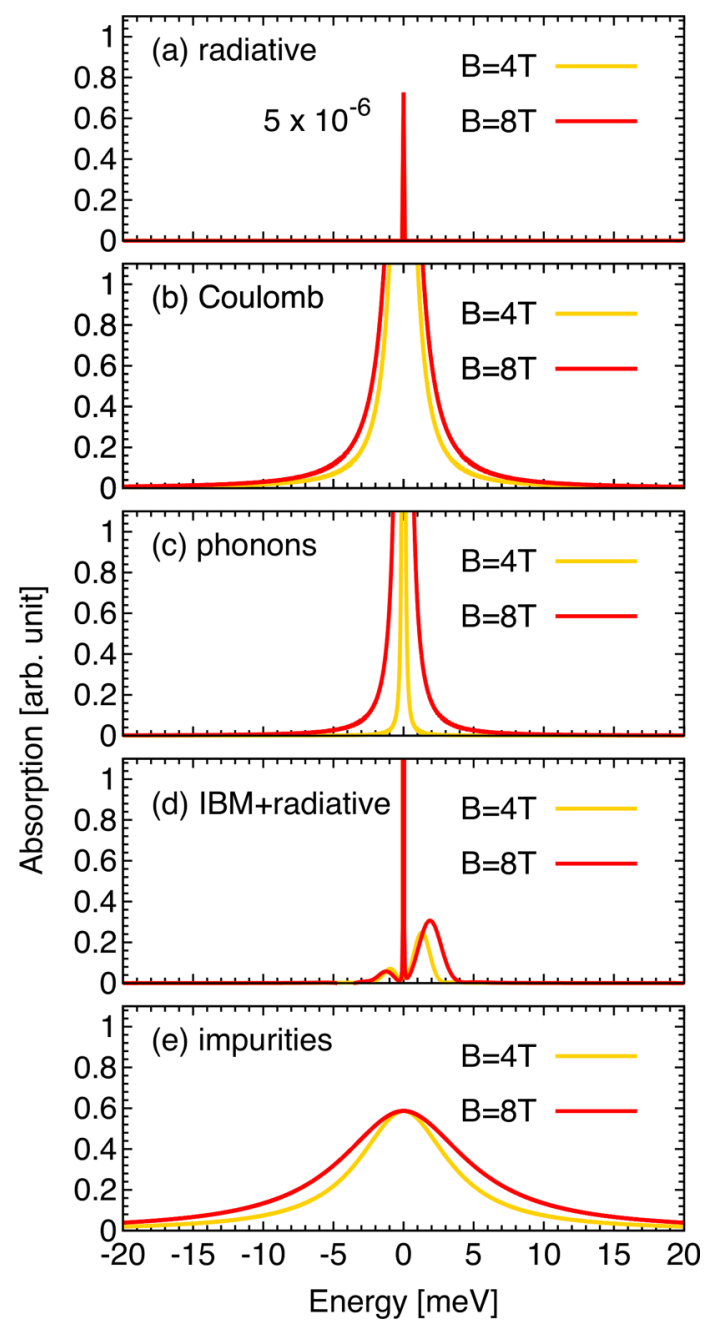

FIG. 2. (Color online) Absorption spectrum for the transition $\mathrm{LL}_{-1} \rightarrow \mathrm{LL}_{+2}$ for different broadening mechanisms: (a) radiative decay, (b) Coulomb scattering, (c) scattering with optical phonons, (d) acoustic phonons (implemented within the IBM with the radiative decay as zero phonon line), and (e) impurity-induced scattering for well separated LLs. Note that the spectrum is spectrally shifted with $\hbar \omega \rightarrow \hbar\left(\omega-\omega_{i j}\right)$.

where the exponent $X^{i j}(t)$ is defined as

$$
X^{i j}(t)=i\left(\omega_{i j}-\Delta^{i j}\right) t+i R_{2}^{i j}(t)-S_{T}^{i j}+R_{1}^{i j}(t)-\gamma t .
$$

It is determined by

$$
\begin{gathered}
\Delta^{i j}=-\sum_{\mathbf{q} \kappa} G_{i j}^{\mathbf{q}} \omega_{\mathbf{q} \kappa}, \quad S_{T}^{i j}=\sum_{\mathbf{q} \kappa} G_{\mathbf{q}}^{i j}\left(2 n_{\mathbf{q} \kappa}+1\right), \\
R^{i j}(t)=\sum_{\mathbf{q} \kappa} G_{\mathbf{q}}^{i j}\left[\left(n_{\mathbf{q} \kappa}+1\right) e^{-i \omega_{\mathbf{q} \kappa} t}+n_{\mathbf{q} \kappa} e^{i \omega_{\mathbf{q} \kappa} t}\right] .
\end{gathered}
$$

The prefactor of $\alpha_{ \pm}^{\text {IBM }}$ includes the summation over the Kronecker $\delta$ 's expressing the optical selection rules, as in Eq. (24). The relaxation of the polarized material due to acoustic phonons, i.e., the electron-phonon interaction, leads to the characteristic IBM exponent $X^{i j}(t)$, which is discussed in detail in Ref. [38]. Furthermore, $n_{\mathbf{q} \kappa}$ is the thermal Bose-Einstein distribution introducing a temperature dependence, while the parameter $G_{\mathbf{q}}^{i j}=\left|g_{\mathbf{q}}^{i i}-g_{\mathbf{q}}^{j j}\right|^{2} /\left(\hbar^{2} \omega_{\mathbf{q} \kappa}^{2}\right)$ is determined by the linear dispersion of the acoustic phonons $\omega_{\mathbf{q} \kappa}=v_{\mathrm{F}}|\mathbf{q}|$ and the diagonal electron-phonon matrix elements $g_{\mathbf{q}}^{i i}$, cf. Eq. (14), which contain the associated Laguerre polynomials giving rise to a characteristic shape of the acoustic phonon-induced broadening, as will be discussed below in Fig. 2.

To be able to evaluate the integral in Eq. (26), a decay rate $\gamma$ was inserted. Since the radiative decay is always present, we assume that its value is determined by $\Gamma_{i j}^{\mathrm{rad}} / \hbar$; cf. Eq. (34). The constant $\Delta^{i j}$ describes a shift in energy, the so-called polaron shift, occurring when polarized graphene interacts with an electron, which itself caused the polarization [41,42]. The Huang-Rhys factor $S_{T}^{i j}$ determines the strength of the coupling with the lattice [43] and therefore the strength of phonon-induced damping, while the time dependence and the weight of phonon emission/absorption is inherent to the dynamics of $R^{i j}(t)$.

\section{BROADENING MECHANISMS}

In this section, we examine the microscopic mechanisms giving rise to Landau level broadening in graphene. We study the absorption spectrum focusing on the broadening of interband absorption peaks. To be able to understand the experimentally observed fixed number of resolvable LLs [14], we investigate the dependence of the broadening on the LL index $n$ and the external magnetic field $B$.

Figure 2 shows the absorption spectrum characterized by a pronounced peak stemming from the LL transition $\mathrm{LL}_{-1} \rightarrow$ $\mathrm{LL}_{+2}$. We show separately different broadening mechanisms for two external magnetic fields $B$. In all cases, an increase of $B$ gives rise to an enhanced energy broadening. We find that the impurity-induced broadening presents the dominant contribution reflecting well the experimentally observed LL broadening [14] in the range of $5 \mathrm{meV}$. In the following, the different broadening mechanisms are discussed in detail.

\section{A. Impurity-induced broadening}

Here, we investigate the Landau level broadening stemming from the scattering of electrons with impurities. An expression for the broadening is obtained within the self-consistent Born approximation $[4,31,32]$. To determine the impurity-induced broadening, we distinguish between two different regimes: (i) the well separated LL regime, where the broadening is smaller than the inter-Landau level spacing and (ii) the densely spaced LL regime with a strong LL overlap.

Figure 3(a) shows the density of states at an external magnetic field of $B=4 \mathrm{~T}$ for a constant impurity broadening of $3.5 \mathrm{meV}$ (corresponding to the experimentally observed value [14]). The lowest 7 LLs have no overlap and are hence well separated. Then, the spectral overlap gradually increases. For $n= \pm 12(n= \pm 17)$, we have an overlap of $10 \%(20 \%)$. In this regime, it is a good approximation to calculate the carrier-impurity scattering assuming the linear electronic band structure of graphene in the absence of a magnetic field [31].

\section{Well separated LL regime}

The impurity broadening for well separated LLs can be obtained within the self-consistent Born approximation by two 

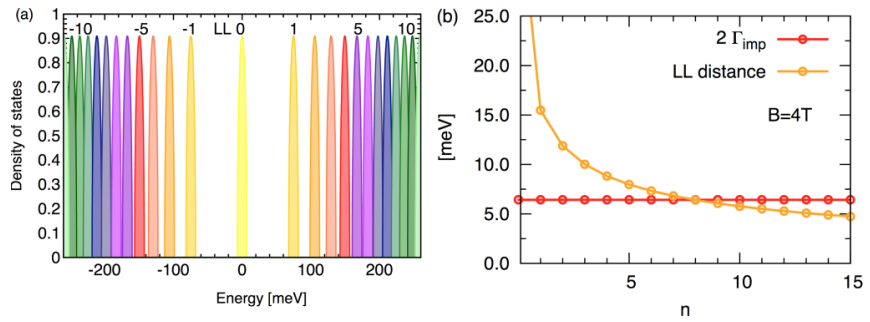

FIG. 3. (Color online) (a) Density of states of the energetically lowest Landau levels as obtained in a self-consistent Born approximation for a constant broadening of $3.5 \mathrm{meV}$ and corresponding impurity scattering parameter of $A_{\mathrm{imp}}=255$. (b) Broadening of LL due to impurities for well separated LLs in comparison to the inter-Landau level energy spacing. A crossing is observed at $n=7$, where LLs start to overlap.

different methods. The first method exploits the Heisenberg equation of motion making use of the impurity Hamiltonian [Eq. (17)] within the density matrix formalism [4]. Hereby the broadening of an absorption peak, i.e., the width of the Lorentzian in Eq. (24), can be directly calculated. The second method is based on Green's functions and yields the density of states [31]:

$$
\operatorname{DOS}(E)=\frac{1}{\pi^{2} v_{F}} \sqrt{\frac{\hbar A_{\mathrm{imp}}}{2 e_{0} B}} \sqrt{1-\frac{A_{\mathrm{imp}}\left(E-E_{n}\right)^{2}}{2\left(\hbar \omega_{c}\right)^{2}}},
$$

which is illustrated in Fig. 3(a). The density of states drops to zero for each LL at $E=E_{n} \pm \hbar \omega_{c} \sqrt{2 / A_{\text {imp }}}$; therefore, the full width at half maximum of each $\mathrm{LL}$ is given by $\Gamma_{\mathrm{FWHM}}^{\mathrm{DOS}}=$ $\hbar \omega_{c} \sqrt{2 / A_{\text {imp }}}$ and yields a Lorentzian broadening of

$$
\Gamma_{\mathrm{imp}}^{\mathrm{well}}=\frac{1}{2} \hbar \omega_{c} \sqrt{\frac{2}{A_{\mathrm{imp}}}}=v_{F} \sqrt{\frac{\hbar e_{0} B}{A_{\mathrm{imp}}}} .
$$

The equation is in accordance with the density matrix-based approach described in Ref. [4], if neglecting the small influence of the form factor. Note that an exact calculation of the density of states yields a Gaussion shape [44]. Comparing the exact result with that of the self-consistent Born approximation, we find a good agreement, in particular with respect to the level broadening [45].

Being proportional to $\sqrt{B}$, the broadening shows the same dependence on the magnetic field as the Landau level energies. As a consequence, the number of well separated Landau levels is constant. Nevertheless, this does not explain the constant number of resolvable LLs for arbitrary magnetic fields, as observed in the experiment [14]. The reason is that partly overlapping LLs can still be resolved if they are sufficiently far apart in energy, and this energy difference increases with the magnetic field; cf. the bright violet lines in Fig. 4.

Note that the same Landau level broadening (except for the form factor) is also obtained for a conventional twodimensional electron gas [29]. However, in this case the Landau level energies scale linearly with $B$, therefore, the Landau level spacings increase faster with the magnetic field than the broadening does. Furthermore, due to the constant Landau level spacings, all LLs are resolvable above a critical magnetic field.

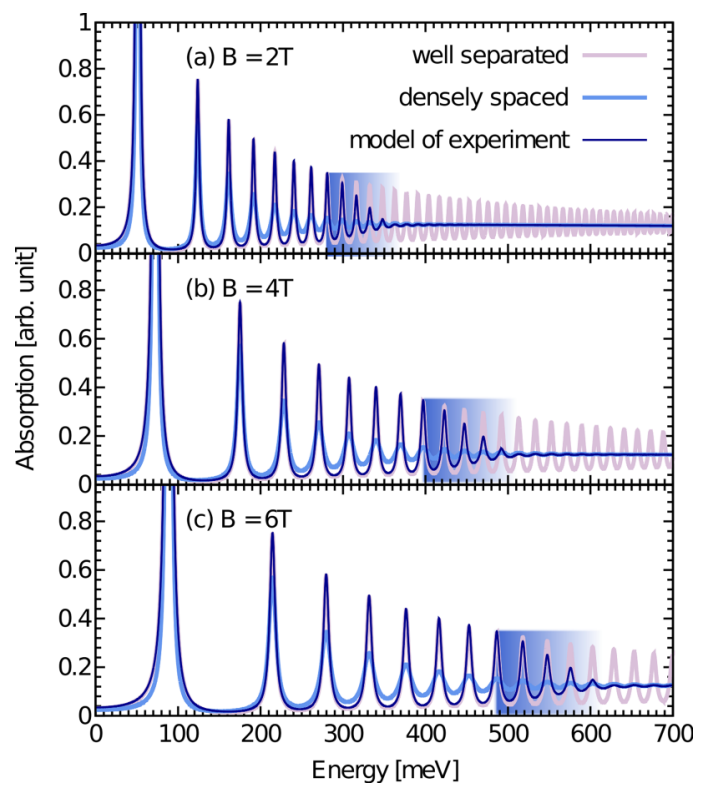

FIG. 4. (Color online) Absorption spectra for different external magnetic fields focusing on the impurity-induced broadening within the regime for well-separated LLs, densely spaced LLs, and a combination of both. The blue-shaded area shows the transition region between the two regimes; cf. the explanation in the text. The investigated conditions correspond to a temperature of $2 \mathrm{~K}$ and a magnetic field strength of $4 \mathrm{~T}$ according to the experimental realization [14].

\section{Densely spaced $L L$ regime}

To calculate the impurity-induced broadening in the regime of densely spaced LLs the self-consistent Born approximation is used. The LL quantization is neglected to calculate the relaxation time, which determines the full width at half maximum of the absorption [31]. The magnetic field then enters via the insertion of the LL energy and we can write for the broadening [32]

$$
\Gamma_{i, j}^{\text {densely }}=\frac{\pi\left|\varepsilon_{i}-\varepsilon_{j}\right|}{A_{\text {imp }}} \sim \sqrt{B}(\sqrt{n+1}+\sqrt{n}) .
$$

The increase of the broadening with $\sqrt{B}(\sqrt{n+1}+\sqrt{n})$ leads to a limited number of resolvable Landau level transitions independently of the magnetic field (cf. the bright blue lines in Fig. 4), as observed in a recent experiment [14]. However, the densely spaced LL regime is not applicable to the low-energetic LLs, which do not overlap; cf. Fig. 3. Therefore, we need to take into account both LL regimes considering a transition region weighted by the overlap of LLs.

\section{Combined approach}

Comparing the inter-Landau level spacings $\hbar \omega_{c}(\sqrt{n+1}-\sqrt{n})$ with the impurity-induced broadening $\Gamma_{\text {imp }}^{\text {well }}$ [Eq. (30)] gives the condition when the LLs start to overlap

$$
(\sqrt{|n+1|}-\sqrt{|n|})<2 \sqrt{\frac{2}{A_{\text {imp }}}} .
$$


Adjusting the carrier-impurity scattering strength of $A_{\text {imp }} \approx$ 255 to the experimentally observed LL broadening of $3.5 \mathrm{meV}$ at $B=4 \mathrm{~T}$ and $T=2 \mathrm{~K}$, we find that at these conditions, LLs are strictly separated for LLs up to $n=7$; cf. Fig. 3(b). This is the valid region for the impurity model addressing the well separated LL regime; cf. Eq. (30). Energetically higher LLs show a gradually increasing overlap counteracting the energy quantization. To develop a model covering all LLs, we introduce a gradual transition (weighted by the LL overlap) from the well-separated regime characterized by zero overlap (up to $n=7$ ) to the densely spaced regime. We assume that for LL $n=12$ with an overlap of approximately $10 \%$ the latter regime has taken over.

Figure 4 shows absorption spectra for three different external magnetic fields directly comparing the results from the model valid in the well separated LL regime, densely spaced LL regime, and the model combining both regimes. We find that the combined model (dark blue line) for the impurity-induced broadening reproduces well the experimental observation that independent of the magnetic field a constant number of approximately 10 resolvable LLs can be found.

\section{B. Radiative broadening}

The most fundamental relaxation process out of an excited state is spontaneous emission, which is also known as radiative decay. Since the process is very slow, it results in a sharp peak in the absorption spectrum, cf. Fig 2(a), which can be calculated using Fermi's golden rule.

Considering the Heisenberg equation for $H_{0, \text { el }}$ and the interaction with a quantized light field $H_{\mathrm{el}-\mathrm{pt}}^{q m}$, the radiative decay rate can be written as [46]

$$
\Gamma_{i j}^{\mathrm{rad}}=2 \pi \hbar \sum_{\mathbf{q}, \sigma}\left|M_{\mathbf{q}, \sigma}^{i j}\right|^{2} \delta\left(\omega_{0}-\omega_{\mathbf{q}}\right) .
$$

Considering the photon dispersion $\omega_{\mathbf{q}}=|\mathbf{q}| c$ with the photon wave vector $\mathbf{q}$ and assuming the cavity volume $V$ to be infinite, we convert the sum into an integral and obtain

$$
\begin{aligned}
\Gamma_{i j}^{\mathrm{rad}}= & \left(\alpha_{n_{i}} \alpha_{n_{j}} e_{0} v_{F}\right)^{2} \frac{\omega_{c}}{16 \pi \varepsilon_{0}}\left(\frac{n_{g}}{c}\right)^{3}\left|\lambda_{i} \sqrt{n_{i}}-\lambda_{j} \sqrt{n_{j}}\right| \\
& \times \delta_{\xi_{i}, \xi_{j}} \delta_{m_{i}, m_{j}}\left(\delta_{n_{i}, n_{j}+1}+\delta_{n_{i}, n_{j}-1}\right),
\end{aligned}
$$

where the optical matrix element $M_{\mathbf{q}, \sigma}^{i j}$ from Eq. (9) was inserted. Here, a correction of the vacuum light velocity $c \rightarrow$ $c / n_{b g}$, with the background refractive index $n_{b g}=\sqrt{\varepsilon_{b g}}$, was introduced, since the screening by the background material is relevant for the radiative decay rate. Assuming the graphene sample to be supported on one side, the dielectric background constant reads $\varepsilon_{b g}=\left(\varepsilon_{\text {air }}+\varepsilon_{\text {substrat }}\right) / 2 \approx 3.3$ for $\operatorname{SiC}$ [37].

We find that the radiative decay rate for Landau-quantized graphene is in the range of $10^{-1} \mu \mathrm{eV}$ yielding a relaxation time on a nanosecond time scale due to spontaneous emission. Concretely, for the transition $\mathrm{LL}_{-1} \rightarrow \mathrm{LL}_{+2}$, it takes approximately $31 \mathrm{~ns}$ to relax back into the initial position. Even though the radiative decay rate [Eq. (34)] exhibits the required dependence on $\sqrt{n B}$ to be able to explain the experimental observation [14], it is negligible for the LL broadening, since
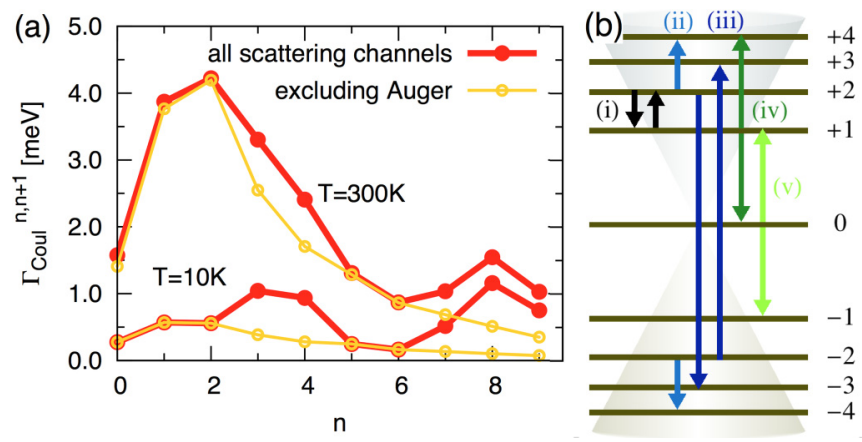

FIG. 5. (Color online) (a) Coulomb-induced LL broadening as a function of the transition $\mathrm{LL}_{-n} \rightarrow \mathrm{LL}_{n+1}$ for two different temperatures with a constant external magnetic field $B=4 \mathrm{~T}$. The comparison between all Coulomb scattering channels (red line) and the case excluding Auger scattering (yellow line) illustrates the outstanding importance of the latter. (b) Schematic illustration of possible Coulomb-induced scattering channel including (i) pure dephasing, (ii) intra- and (iii) interband scattering, (iv) Auger, and (v) Auger-like scattering. Reverse processes are not shown.

it is too small. Nevertheless, radiative decay is important as the initial broadening for other mechanisms; cf. Sec. III D 2.

\section{Coulomb-induced broadening}

The Coulomb interaction influences the LL broadening via the time-dependent many-particle scattering rates $[14,47]$ :

$$
\Gamma_{i j}^{\text {Coul }}=\frac{\hbar}{2} \sum_{l=i, j}\left[S_{l, C}^{\text {in }}(t)+S_{l, C}^{\text {out }}(t)\right]
$$

that enter the Bloch equation for the occupation probability [Eq. (21)] and read [13]

$$
\begin{gathered}
S_{l, C}^{\mathrm{in}}(t)=\frac{2 \pi}{\hbar} \sum_{\mathrm{abc}} V_{b c}^{l a} \tilde{V}_{b c}^{l a}\left(1-\rho_{a}\right) \rho_{b} \rho_{c} L_{\Gamma}\left(\Delta E_{b c}^{l a}\right), \\
S_{l, C}^{\text {out }}(t)=\frac{2 \pi}{\hbar} \sum_{\mathrm{abc}} \tilde{V}_{b c}^{l a} \rho_{a}\left(1-\rho_{b}\right)\left(1-\rho_{c}\right) L_{\Gamma}\left(\Delta E_{b c}^{l a}\right) .
\end{gathered}
$$

They are determined by the carrier occupations $\rho_{l}$, the Coulomb matrix elements $\tilde{V}_{b c}^{l a}=V_{b c}^{l a}\left(V_{l a}^{b c}-V_{l a}^{c b}\right)$, the energy difference $\Delta E_{\mathrm{labc}}=\varepsilon_{l}+\varepsilon_{a}-\varepsilon_{b}-\varepsilon_{c}$, and the Lorentzian $L_{\Gamma}(E) \frac{\Gamma_{L}}{\pi\left(\Delta E^{2}+\Gamma_{L}^{2}\right)}$. The latter expresses the energy conservation, where the broadening $\Gamma_{L}$ is considered to be induced by the impurity scattering $\Gamma_{\text {imp }}$.

Figure 5(a) shows Coulomb-induced LL broadenings at $B=4 \mathrm{~T}$ in dependence of the interband transition $\mathrm{LL}_{-n} \rightarrow$ $\mathrm{LL}_{n+1}$ for different temperatures $T$. We find a strong dependence on both $n$ and $T$. For energetically low LLs $n=1,2$, we obtain broadenings in the range of $4 \mathrm{meV}(0.5 \mathrm{meV})$ for $300 \mathrm{~K}(10 \mathrm{~K})$. The broadening is given by the sum of the incoming and outgoing scattering rates of the two involved LLs of a given transition $\mathrm{LL}_{-n} \rightarrow \mathrm{LL}_{n+1}$ [Eq. (35)]. Each of these scattering rates $S_{l}^{\text {in/out }}$ depends on three carrier occupations $\rho_{a}$, $\rho_{b}, \rho_{c}$ and incorporates a Lorentzian. While every summand of Eq. (36) describes an energy-conserving scattering event of two electrons from the initial states $a$ and $b$ to the final states $c$ and $l$, Eq. (37) incorporates the sum of the reverse processes, 
respectively. As a result, Pauli blocking plays an important role for Coulomb-induced scattering processes.

In order to explain the dependence of the LL broadening on the transition $\mathrm{LL}_{-n} \rightarrow \mathrm{LL}_{n+1}$, we now consider the impact of specific electron-electron scattering channels: (i) pure dephasing processes that do not change the carrier occupation in the involved LLs, (ii) intraband processes involving two electrons that both remain in the same band, (iii) interband processes bridging the valence and the conduction band, (iv) Auger processes that are characterized by one electron changing the band, while the second electron remains in its initial band, and (v) Auger-like processes incorporating two electrons scattering into or out of the zeroth LL (that belongs to both the conduction and the valence band); cf. Fig. 5(b).

In Fig. 5(a), the influence of Auger scattering (iv) is illustrated by directly comparing all Coulomb scattering processes (red line) and only non-Auger processes (yellow line) for two different temperatures $T$. The high impact of $T$ for the energetically lowest LLs can be ascribed to the non-Auger processes that are denoted by (i)-(iii), (v) in Fig. 5(a). Taking into account Auger channels (iv), the difference between the red and the yellow lines remains similar at both investigated temperatures. At low temperatures, apart from the half-filled zeroth LL, all LLs are either empty $\left(\rho_{n \neq 0}^{c}=0\right)$ or full $\left(\rho_{n \neq 0}^{v}=1\right)$ and, as a consequence, Coulomb scattering is completely suppressed via Pauli blocking. Nevertheless, Pauli blocking due to the occupation $\rho_{l}$ in the investigated state $l$ does not enter the scattering rates $S_{l}^{\text {in/out }}$ [Eqs. (36) and (37)] and results in a finite Coulomb-induced broadening even at zero temperature. To give a concrete example, the Auger-like scattering process (v) in Fig. 5(b) is strongly suppressed by Pauli blocking at low temperatures, since $\mathrm{LL}_{-1}$ is completely occupied, and yet the scattering rate $S_{-1}^{\text {in }} \propto \rho_{0} \rho_{0}\left(1-\rho_{1}\right) \neq 0$ yields a contribution to the broadening of all absorption peaks that involve $\mathrm{LL}_{-1}$. Since Auger-like scattering is generally possible between $\mathrm{LL}_{0}$ and $\mathrm{LL}_{ \pm n}$, all $\mathrm{LL}$ transitions are subject to a finite broadening. However, the matrix elements for Auger-type scattering strongly decreases for higher values of $n$, therefore, the broadening clearly decreases with $n$; cf. the yellow line in Fig. 5(a). Likewise, also pure dephasing (i) processes involving the zeroth LL contribute to the broadening at low temperatures. At higher temperatures, the Pauli blocking is generally weakened as carriers are thermally distributed in energetically lower LLs, i.e., $\rho_{1}, \rho_{2}$ are no longer empty. This enables other scattering processes to contribute to the Coulomb-induced broadening. In particular, intraband (ii) as well as interband (iii) scattering becomes feasible. As a consequence, the LL broadening for the energetically lowest LLs clearly increases with the temperature; cf. Fig. 5(a).

Including Auger processes we find that they are responsible for the interesting dependence of the energy broadening on the inter-LL transition $\mathrm{LL}_{-n} \rightarrow \mathrm{LL}_{n+1}$; cf. the red lines in Fig. 5(a). The reason for this exceptional role of Auger processes is the fact that they require equidistant LLs to occur. As we can see in Fig. 5(b), LL $n=1$ is energetically located in the center of $n=0$ and $n=4$ permitting Auger scattering of the type $\mathrm{LL}_{0} \rightarrow \mathrm{LL}_{1}$ in conjunction with $\mathrm{LL}_{4} \rightarrow$ $\mathrm{LL}_{1}$. Following the same line of reasoning as above, we deduce that the nonvanishing scattering rate $S_{4}^{\text {out }}(t) \sim \rho_{0}(1-$ $\left.\rho_{1}\right)^{2} \neq 0$ gives rise to a broadening of inter-LL transitions that incorporate the LL $n=4$. This leads to an increased broadening of the absorption peaks corresponding to the transitions $\mathrm{LL}_{-3} \rightarrow \mathrm{LL}_{4}$ and $\mathrm{LL}_{-4} \rightarrow \mathrm{LL}_{+5}$ due to Auger scattering, which is clearly visible in Fig. 5(a). Similarly, there are more ladders of equidistant Landau levels $n=$ $0,2,8, n=0,3,12$, etc., resulting in a broadening of inter-LL transitions that incorporate the LLs $n=8,12$, etc.; hence an increased broadening of the transitions $\mathrm{LL}_{-7} \rightarrow \mathrm{LL}_{+8}$ and $\mathrm{LL}_{-8} \rightarrow \mathrm{LL}_{+9}$ is observed in Fig. 5(a).

In summary, Coulomb interaction contributes significantly to the LL broadening. However, its strength is too small to explain the experimentally observed broadenings [14] of approximately $5 \mathrm{meV}$ at a temperature of $2 \mathrm{~K}$. Furthermore, its interesting dependence on the LL transition $\mathrm{LL}_{-n} \rightarrow \mathrm{LL}_{n+1}$ has not been observed yet in experiments.

\section{Phonon-induced broadening}

The energy of the acoustic phonons is too small to efficiently induce inter-LL transitions [4]; therefore, they are considered within the pure dephasing inducing non-Markovian independent boson model. On the other hand, the higher energetic optical phonons are capable of efficiently inducing inter-LL transitions, if their energies are in resonance [9]. Consequently, the broadening due to optical phonons is obtained from phonon-induced scattering rates within the second-order Born-Markov approximation [37].

\section{Optical phonons}

The interaction of charge carriers with optical phonons is described by time-dependent scattering rates in analogy to the case of the Coulomb interaction [37]

$$
\Gamma_{i j}^{\text {Ph-op }}=\frac{\hbar}{2} \sum_{l=i, j}\left[S_{l, p h}^{\text {in }}(t)+S_{l, p h}^{\text {out }}(t)\right]
$$

that enter the Bloch equation for the occupation probability [Eq. (21)] and read [13]

$$
\begin{aligned}
S_{l, p h}^{\mathrm{in}}(t)= & \frac{2 \pi}{\hbar} \sum_{i} \sum_{\mathbf{q} \kappa}\left|g_{i l}^{\mathbf{q} \kappa}\right| \rho_{i} \\
& \times\left[\left(n_{\mathbf{q} \kappa}+1\right) L_{\Gamma}\left(\Delta E_{i l \kappa}^{\mathrm{em}}\right)+n_{\mathbf{q} \kappa} L_{\Gamma}\left(E_{i l \kappa}^{\mathrm{ab}}\right)\right], \\
S_{l, p h}^{\text {out }}(t)(t)= & \frac{2 \pi}{\hbar} \sum_{i} \sum_{\mathbf{q} \kappa}\left|g_{l i}^{\mathbf{q} \kappa}\right|\left(1-\rho_{i}\right) \\
& \times\left[\left(n_{\mathbf{q} \kappa}+1\right) L_{\Gamma}\left(\Delta E_{l i \kappa}^{\mathrm{em}}\right)+n_{\mathbf{q} \kappa} L_{\Gamma}\left(E_{l i \kappa}^{\mathrm{ab}}\right)\right] .
\end{aligned}
$$

Here, $n_{\mathbf{q} \kappa}$ is the phonon occupation that is determined by the Bose-Einstein distribution. Furthermore, $E_{j i \kappa}^{\mathrm{em} / \mathrm{ab}}=\varepsilon_{i}-$ $\varepsilon_{j} \pm \varepsilon_{\mathbf{q} \kappa}$ is the energy difference for the emission (+) and absorption (-) of an optical phonon with the energy $\varepsilon_{\mathbf{q} \kappa}$. While phonon absorption requires a finite temperature $T$, since the corresponding rates are proportional to $n_{\mathbf{q} \kappa}$, the emission of phonons is also possible at $T=0 \mathrm{~K}$ due to the dependence on $\left(n_{\mathbf{q} \kappa}+1\right)$. Therefore, we focus our discussion here on phonon emission processes.

The impact of the optical phonons on the broadening is determined by resonances that occur between inter-LL transitions and the energy of the involved optical phonon; cf. 

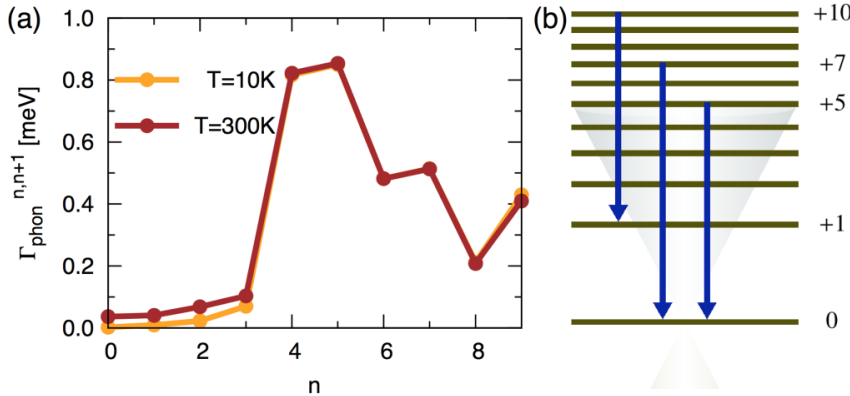

FIG. 6. (Color online) (a) Energy broadening induced by optical phonons as a function of the transition $\mathrm{LL}_{-n} \rightarrow \mathrm{LL}_{n+1}$ for two different temperatures at a constant external magnetic field $B=4 \mathrm{~T}$. (b) Schematic illustration of the underlying most important phonon-induced transitions $\mathrm{LL}_{10} \rightarrow \mathrm{LL}_{1}, \mathrm{LL}_{7} \rightarrow \mathrm{LL}_{0}$, and $\mathrm{LL}_{5} \rightarrow \mathrm{LL}_{0}$.

Fig. 6. For optical phonons, we assume constant energies with $\varepsilon_{\Gamma \mathrm{TO}}=192 \mathrm{meV}, \varepsilon_{\Gamma \mathrm{LO}}=198 \mathrm{meV}, \varepsilon_{\mathrm{KTO}}=162 \mathrm{meV}$, and $\varepsilon_{\mathrm{KLO}}=151 \mathrm{meV}[13,48]$. Since the carrier-phonon coupling of the KLO mode is much smaller than that of the other modes (cf. Sec. II A), we only consider resonances between inter-LL transitions and the phonon modes ГTO, ГLO, and KTO.

At a magnetic field of $4 \mathrm{~T}$, the transitions $\mathrm{LL}_{10} \rightarrow$ $\mathrm{LL}_{1}, \mathrm{LL}_{7} \rightarrow \mathrm{LL}_{0}$, and $\mathrm{LL}_{5} \rightarrow \mathrm{LL}_{0}$ are in approximate resonance with the dominant optical phonon modes. Similar to the Coulomb-induced broadening, the occupation $\rho_{l}$ does not enter the scattering rates $S_{l, p h}^{\text {in/out}}$; hence also scattering events that are suppressed by Pauli blocking contribute to the energy broadening. Therefore, the scattering rates for the above transitions are nonzero and induce a broadening of the absorption peaks involving LLs with the indices $n_{i}=$ $5,7,10$. Consequently, the increased broadenings of LLs $n=$ 4,5 can be ascribed to the phonon-induced transition $\mathrm{LL}_{5} \rightarrow$ $\mathrm{LL}_{0}$. Similarly, the contribution to the broadening of the LLs $n=6,7(9,10)$ stems from the transition $\mathrm{LL}_{7} \rightarrow \mathrm{LL}_{0}\left(\mathrm{LL}_{10} \rightarrow\right.$ $\left.\mathrm{LL}_{0}\right)$; cf. Fig. 6. The observed temperature dependence is very weak, because the energy $k_{\mathrm{B}} T$ at room temperature is not high enough to thermally excite the optical phonon modes.

The strength of the phonon-induced broadening is in the range of $1 \mathrm{meV}$ for $\mathrm{LL}$ transitions fulfilling the resonance conditions with optical phonon energy. Larger broadenings can be achieved for resonant optically allowed LL transitions, since the form factors appearing in Eqs. (14)-(16) favor such transitions [9].

\section{Acoustic phonons}

The broadening due to acoustic phonons does not contribute to the LL broadening through a Lorentzian in the Bloch equations, but as a complex function that is determined by the Huang-Rhys factor $S_{T}$, the dynamics inherent to $R(t)$, and the polaron shift $\Delta$; cf. Eq. (26). Figure 7 illustrates the absorption spectrum explicitly including the acoustic phononinduced LL broadening and its dependence on the transition $\mathrm{LL}_{n+1} \rightarrow \mathrm{LL}_{-n}$ and the temperature $T$. The polaron shift $\Delta$, i.e., the energetic shift with respect to the zero-phonon line is very small. For $B=4 \mathrm{~T}$ and the transition $\mathrm{LL}_{+2} \rightarrow \mathrm{LL}_{-1}$,

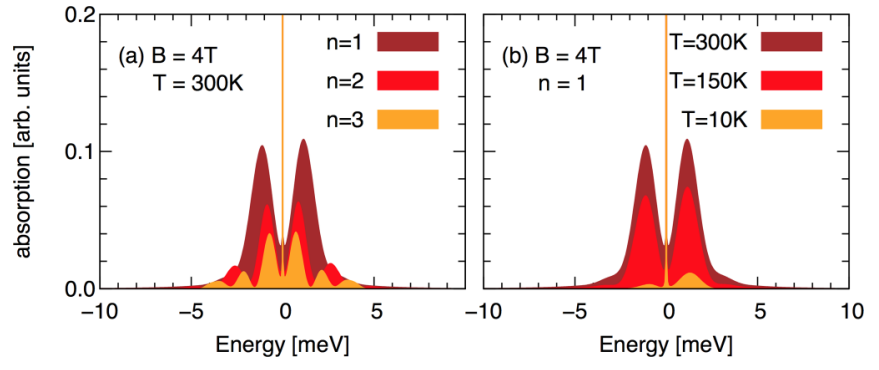

FIG. 7. (Color online) Absorption spectrum considering the broadening due to acoustic phonons at a constant external magnetic field of $4 T$ for (a) different transitions $\mathrm{LL}_{n+1} \rightarrow \mathrm{LL}_{-n}$ at a constant temperature $T=300 \mathrm{~K}$ and for (b) different temperatures considering the constant transition $\mathrm{LL}_{+2} \rightarrow \mathrm{LL}_{-1}$.

it is found to be $16.2 \mu \mathrm{eV}$. The nonlinear dynamics enters through the time-dependent factor $R(t)$. It gives weight to absorption and emission with complex terms proportional to $n_{\mathbf{q}}$ and $\left(n_{\mathbf{q}}+1\right)$, respectively. The strength of the coupling with acoustic phonons is determined by the temperature-dependent Huang-Rhys factor $S_{T}=R(t=0)$.

To be able to numerically calculate the Fourier transformation in Eq. (26), we have included the radiative broadening Eq. (34). In Fig. 7, we can identify the very slow radiative decay as the energetically sharp zero-phonon line. The temperaturedependent sidebands are caused by the emission $\left(E-E_{0}>0\right)$ and absorption $\left(E-E_{0}<0\right)$ of acoustic phonons. Their frequency range is continuous owing to the linear dispersion $\omega_{\mathbf{q}, \mathrm{LA}}$.

The strength of the broadening due to acoustic phonons depends on the external magnetic field (cf. Fig. 2) and increases for higher fields since Eq. (26) is proportional to $\sqrt{B}$. Figure 7 (a) shows that the number of sidebands is proportional to the LL number $n$, while the full width of the absorption is approximately constant. This relation is introduced by the factor $G_{i j}^{\mathbf{q}} \sim\left|g^{i i}-g^{j j}\right|$, which contains the Laguerre polynomials of the form factor from Eq. (12).

Figure 7(b) shows the absorption of the transition $\mathrm{LL}_{+2} \rightarrow$ $\mathrm{LL}_{-1}$ for a constant magnetic field at different temperatures $T$. For small $T$, the emission of phonons is predominant. Increasing the temperature, the occupation of phonons $n_{\mathbf{q} \kappa}$ becomes larger and leads to an enhanced absorption of acoustic phonons and more pronounced side peaks. This can be traced back to the temperature dependence of the Bose-Einstein distribution $n_{\mathbf{q} \kappa}$ appearing in Eqs. (26) and (28).

The characteristic line shape of the acoustic phonon broadening is difficult to quantify. We approximate the FWHM for the LL $n=1$ and the temperature $T=10 \mathrm{~K}$ to be about $1 \mathrm{meV}$. The higher the temperature, the larger the FWHM becomes. In summary, the experimentally observed fixed number of resolvable Landau levels cannot be explained with the broadening contribution of acoustic or optical phonons.

\section{DISCUSSION}

Now, we compare the different mechanisms contributing to the Landau level broadening including radiative decay, Coulomb scattering, optical phonon scattering, and 


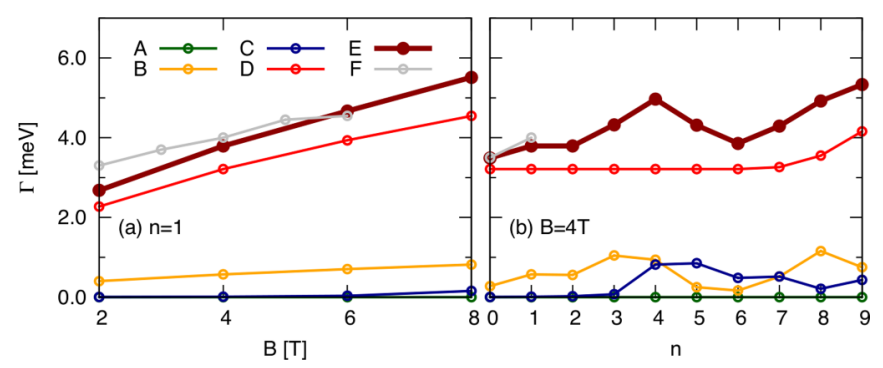

FIG. 8. (Color online) Landau level broadening induced by radiative decay (A), Coulomb scattering (B), optical phonon scattering (C), impurities for $A_{\text {imp }}=255$ (D), the sum of all contributions (E), and the experimental data $(\mathrm{F})$ is shown at a constant temperature of $T=2 \mathrm{~K}$ as a function of (a) the external magnetic field $B$ for the fixed transition $\mathrm{LL}_{-1} \rightarrow \mathrm{LL}_{+2}$ and as a function of (b) the transition $\mathrm{LL}_{-n} \rightarrow \mathrm{LL}_{n+1}$ for the fixed magnetic field $B=4 \mathrm{~T}$.

carrier-impurity scattering. Figure 8 exhibits the dependence of these broadening mechanisms on the magnetic field $B$ and the LL index $n$. Experimental data obtained by Orlita et al. [14] is also shown. The broadening induced by acoustic phonons is calculated as a non-Markovian process via the IBM, and is not included in Fig. 8, since the IBM broadening follows a more complex pattern and does not obey a simple Lorentzian line shape. However, we can estimate it to be smaller than $0.5 \mathrm{meV}$ for small temperatures; cf. Fig. 7(b). In fact, the acoustic phonon-induced broadening becomes relevant only at higher temperatures, where it reaches values of several $\mathrm{meV}$. Furthermore, in order to observe the predicted LL splitting, the other broadening mechanisms have to be strongly suppressed. This can be achieved, e.g., by using a very clean graphene sample (small impurity-induced broadening) that is placed in a strong dielectric material considerably reducing the Coulomb interaction via screening, and using a magnetic field that prevents resonances between optical phonons and inter-LL transitions.

The sum of all relevant broadening mechanisms at low temperatures shows a good qualitative agreement with the experimental values; cf. lines $\mathrm{E}$ and $\mathrm{F}$ in Fig. 8. The LL broadening shows a $\sqrt{B}$ dependence on the magnetic field. The value of $\Gamma=2.75 \mathrm{meV}$ at $B=2 \mathrm{~T}$ is doubled when increasing the field to $B=8 \mathrm{~T}$; cf. Fig. 8(a). The main contribution stems from electron-impurity scattering, which is also the crucial part to explain the experimentally observed constant number of resolvable LLs; cf. Fig. 4. This can be ascribed to the impurity-induced broadening in the densely spaced LL regime resulting in an increase of $\Gamma$ with $n$ that starts to have an impact at $n=7$; cf. line D in Fig. 8(b). Note that only impurity-induced scattering can explain the observed level broadening (the other broadening mechanisms yield a much smaller broadening) and the observed constant number of resolvable LLs. While all broadening mechanisms show an increase of $\Gamma$ with the magnetic field [Fig. 8(a)], the $n$ dependence is more distinguished [cf. Fig. 8(b)]. Unfortunately, only the values for two different inter-LL transitions could be extracted from the experiment. Our calculations clearly attribute the observed increase of the broadening of the energetically lowest LLs to the Coulomb scattering; cf. the lines B and E in Fig. 8(b). We further predict a nonmonotonous dependence of the broadening on the LL index $n$ due to the efficient Auger channels that sensitively depend on $n$. Their contribution can be distinguished from the optical phonon contribution by varying the magnetic field: while Auger scattering explicitly depends on $n$, the dependence of optical phonon scattering on $n$ is due to magnetophonon resonances that occur at different $n$ for different magnetic fields. Experimental values for higher transitions would be desirable to test these predictions.

In summary, we have calculated the energy broadening mechanisms in Landau-quantized graphene including radiative coupling, carrier-carrier, carrier-phonon, and carrier-impurity scattering. We reveal that the radiative broadening is identified to be very small, while the Coulomb- and phonon-induced broadening shows an interesting dependence on the Landau level $n$. We find that only the carrier-impurity scattering with its square-root dependence on the magnetic field $B$ and the LL index $n$ can explain the experimental observation that a constant number of Landau levels can be resolved independently of the applied magnetic field.

\section{ACKNOWLEDGMENTS}

We acknowledge financial support from the Deutsche Forschungsgemeinschaft through SPP 1459 and SFB 910 (HF). Furthermore, E.M. is thankful to the Swedish Research Council (VR) and the EU Graphene Flagship (CNECT-ICT604391).
[1] K. S. Novoselov, A. K. Geim, S. V. Morozov, D. Jiang, M. I. Katsnelson, I. V. Grigorieva, S. V. Dubonos, and A. A. Firsov, Nature (London) 438, 197 (2005).

[2] Y.Zhang, Y.-W. Tan, H. L. Stormer, and P. Kim, Nature (London) 438, 201 (2005).

[3] M. O. Goerbig, Rev. Mod. Phys. 83, 1193 (2011).

[4] F. Wendler, A. Knorr, and E. Malic, Nat. Commun. 5, 3703 (2014).

[5] M. Mittendorff, F. Wendler, E. Malic, A. Knorr, M. Orlita, M. Potemski, C. Berger, W. A. de Heer, H. Schneider, M. Helm, et al., Nat. Phys. 11, 75 (2014).

[6] F. Wendler and E. Malic, Sci. Rep. 5, 12646 (2015).
[7] P. Plochocka, P. Kossacki, A. Golnik, T. Kazimierczuk, C. Berger, W. A. de Heer, and M. Potemski, Phys. Rev. B 80, 245415 (2009).

[8] W.-P. Li, J.-W. Yin, Y.-F. Yu, and Z.-W. Wang, Solid State Commun. 163, 19 (2013).

[9] F. Wendler, A. Knorr, and E. Malic, Appl. Phys. Lett. 103, 253117 (2013).

[10] Z.-W. Wang, L. Liu, L. Shi, X.-J. Gong, W.-P. Li, and K. Xu, J. Phys. Soc. Jpn. 82, 094606 (2013).

[11] M. Mittendorff, M. Orlita, M. Potemski, C. Berger, W. A. de Heer, H. Schneider, M. Helm, and S. Winnerl, New J. Phys. 16, 123021 (2014). 
[12] Z.-W. Wang, L. Liu, and Z.-Q. Li, Europhys. Lett. 108, 36005 (2014).

[13] F. Wendler, A. Knorr, and E. Malic, Nanophotonics 4, 224 (2015).

[14] M. Orlita, C. Faugeras, R. Grill, A. Wysmolek, W. Strupinski, C. Berger, W. A. de Heer, G. Martinez, and M. Potemski, Phys. Rev. Lett. 107, 216603 (2011).

[15] M. L. Sadowski, G. Martinez, M. Potemski, C. Berger, and W. A. de Heer, Phys. Rev. Lett. 97, 266405 (2006).

[16] D. S. L. Abergel and V. I. Fal'ko, Phys. Rev. B 75, 155430 (2007).

[17] J. Rioux, G. Burkard, and J. E. Sipe, Phys. Rev. B 83, 195406 (2011).

[18] R. C. Jones, J. Opt. Soc. Am. 31, 488 (1941).

[19] K. M. Rao and J. E. Sipe, Phys. Rev. B 86, 115427 (2012).

[20] H. Haken, Quantum Field Theory of Solids (North-Holland, Amsterdam, 1976).

[21] H. Suzuura and T. Ando, Phys. Rev. B 65, 235412 (2002).

[22] E. Mariani and F. von Oppen, Phys. Rev. B 82, 195403 (2010).

[23] E. Mariani and F. von Oppen, Phys. Rev. Lett. 100, 076801 (2008).

[24] K. H. Michel and B. Verberck, Phys. Rev. B 78, 085424 (2008).

[25] E. Malic, T. Winzer, E. Bobkin, and A. Knorr, Phys. Rev. B 84, 205406 (2011).

[26] W.-K. Tse and S. Das Sarma, Phys. Rev. B 79, 235406 (2009).

[27] F. Wendler and E. Malic, Phys. Status Solidi B 251, 2541 (2014).

[28] S. Piscanec, M. Lazzeri, F. Mauri, A. C. Ferrari, and J. Robertson, Phys. Rev. Lett. 93, 185503 (2004).

[29] T. Ando and Y. Uemura, J. Phys. Soc. Jpn. 36, 959 (1974).

[30] T. Ando, J. Phys. Soc. Jpn. 38, 989 (1975).

[31] N. H. Shon and T. Ando, J. Phys. Soc. Jpn. 67, 2421 (1998).

[32] T. Ando, Y. Zheng, and H. Suzuura, J. Phys. Soc. Jpn. 71, 1318 (2002).
[33] E. Akkermans and G. Montambaux, Mesoscopic Physics of Electrons and Photons (Cambridge University Press, Cambridge, UK, 2007).

[34] Y. C. Huang, C. P. Chang, and M. F. Lin, Nanotechnology 18, 495401 (2007).

[35] J. Bai, R. Cheng, F. Xiu, L. Liao, M. Wang, A. Shailos, K. L. Wang, Y. Huang, and X. Duan, Nat. Nanotechnol. 5, 655 (2010).

[36] H. Haug and S. W. Koch, Quantum Theory of the Optical and Electronic Properties of Semiconductors (World Scientific, Singapore, 2009).

[37] E. Malic and A. Knorr, Graphene and Carbon Nanotubes: Ultrafast Optics and Relaxation Dynamics (Wiley-VCH, New York, 2013).

[38] G. D. Mahan, Many-Particle Physics (Plenum Press, New York, 1981).

[39] F. Wendler, H. Funk, M. Mittendorff, S. Winnerl, M. Helm, A. Knorr, and E. Malic, Proc. SPIE 9361, 936105 (2015).

[40] G. C. Wick, Phys. Rev. 80, 268 (1950).

[41] S. Schmitt-Rink, D. A. B. Miller, and D. S. Chemla, Phys. Rev. B 35, 8113 (1987).

[42] G. Pupillo, A. Griessner, A. Micheli, M. Ortner, D.-W. Wang, and P. Zoller, Phys. Rev. Lett. 100, 050402 (2008).

[43] K. Huang and A. Rhys, R. Soc. 204, 406 (1950).

[44] F. Wegner, Z. Phys. B 51, 279 (1983).

[45] T. Dittrich, P. Haengi, G.-L. Ingold, B. Kramer, G. Schoen, and W. Zwerger, Quantum Transport and Dissipation (Wiley-VCH, New York, 1998).

[46] M. O. Scully, Quantum Optics (Cambridge University Press, Cambridge, UK, 1997).

[47] L. Zheng and S. Das Sarma, Phys. Rev. B 53, 9964 (1996).

[48] J. Maultzsch, S. Reich, C. Thomsen, H. Requardt, and P. Ordejón, Phys. Rev. Lett. 92, 075501 (2004). 